\title{
Properties of Caputo-Fabrizio fractional operators
}

\author{
Gustavo Asumu M Boro Nchama
}

Universidad Nacional de Guinea Ecuatorial, Malabo, Guinea Ecuatorial

Received: 7 November 2019, Accepted: 22 December 2019

Published online: 13 February 2020.

\begin{abstract}
In this paper, we have studied some theoretical properties concerning the Caputo-Fabrizio fractional operators. Also we obtained expressions for the fractional integral and derivative of some elementary functions. As auxiliary results, general integration formulas for functions with radicals of any order are presented.
\end{abstract}

Keywords: Caputo-Fabrizio fractional derivative, Caputo-Fabrizio fractional integral, integral of radical functions.

\section{Introduction}

The fractional calculus is a name for the theory of integrals and derivatives of arbitrary order, which unify and generalize the notions of integer-order differentiation and $n$-fold integration. The history of the Fractional Calculus goes back to seventeenth century, when in 1695 the derivative of order $\alpha=1 / 2$ was described by Leibnitz in his letter to L'Hospital [28]. That date is regarded as the exact birthday of the fractional calculus. Since then this branch has been treated by eminent mathematicians, such as Euler, Laplace, Fourier, Liouville, Riemann, Laurent, Weyl and Abel. And therefore many definitions, concerning the fractional operators have been proposed:

Grunwald and Letnikov faced the problem of non-integer differentiation [20], generalizing the derivative definition of an integer order, based on the quotient concept incremental, using the following formula

$$
\left(D^{\alpha} f\right)(x)=\lim _{h \rightarrow 0} \frac{\left(\nabla_{h}^{\alpha} f\right)(x)}{h^{\alpha}}
$$

where

$$
\left(\nabla_{h}^{\alpha} f\right)(x)=\sum_{j=0}^{n}(-1)^{j}\left(\begin{array}{c}
\alpha \\
j
\end{array}\right) f(x-j h)
$$

with $n=[\alpha]$.

In 1917 Weyl [21] defined a fractional integral adequate to periodic functions

$$
I_{+}^{\alpha} f(x)=\frac{1}{\Gamma(\alpha)} \int_{x}^{\infty}(t-x)^{\alpha-1} f(t) d t \quad \operatorname{Re}(\alpha)>0 .
$$

In 1938 M. Riesz published a number of papers [22] which are centered around the integral

$$
{ }^{R} D_{t}^{\alpha} u(t)=\frac{1}{\Gamma(n-\alpha)}\left(\frac{d}{d t}\right)^{n} \int_{a}^{b}|t-\tau|^{n-(\alpha+1)} u(\tau) d \tau
$$

\footnotetext{
*Corresponding author e-mail: asumu@matcom.uh.cu
} 
In [23], Laurent used a contour given as an open circuit (known as Laurent loop) instead of a closed circuit used by Sonin and Letnikov and thus produced today's definition of the Riemann-Liouville fractional integral

$$
D_{a x}^{-\alpha} f(x)=\frac{1}{\Gamma(\alpha)} \int_{a}^{x}(x-t)^{\alpha-1} f(t) d t, \quad \operatorname{Re}(\alpha)>0 .
$$

The Riemann-Liouville derivative appears to the paper by N. Ya. Sonin in [24-25] where he used Cauchy's integral formula as a starting point to reach differentiation with arbitrary index

$$
{ }^{R L} D_{a t}^{\alpha} u(t)=\frac{1}{\Gamma(n-\alpha)}\left(\frac{d}{d t}\right)^{n} \int_{a}^{t}(t-s)^{n-(\alpha+1)} u(s) d s
$$

Consequently, M. Caputo proposed the following new definition of fractional derivative

$$
{ }^{C} D_{0 t}^{\alpha} u(t)=\frac{1}{\Gamma(n-\alpha)} \int_{0}^{t}(t-s)^{n-(\alpha+1)} u^{(n)}(s) d s .
$$

Recently Caputo and Fabrizio launched a new fractional derivative and it was followed by some related theoretical and applied results [26]. The interest for this new approach is due to the necessity to describe material heterogeneities and structures with different scales, which cannot be well described by classical local theories [26].

In the last few decades many authors pointed out that derivatives and integrals of non-integer order are very suitable for the description of properties of various real materials. It has been shown that new fractional-order models are more adequate that integer-order models [27]. Fractional derivatives provide an excellent instrument for the description of memory and hereditary properties of various materials and processes. This is the main advantage of fractional derivatives in comparison with classical integer-order models, in which such effects are in fact neglected. The advantages of fractional derivatives become apparent in modelling mechanical and electrical properties of real materials, as well as in the description of rheological properties of rocks, and in market behaviour [27, 30].

The other large field which requires the use of derivatives of non-integer order is the theory of fractals. The development of the theory of fractals has opened further perspectives for the theory of fractional derivatives, especially in modelling dynamical processes in self-similar and porous structures [27].

In recent years, many researcher have shown that Fractional Calculus is a useful tool in image processing field such as image enhancement, image denoising, image edge detection, image segmentation, image registration, image recognition, image fusion, image encryption, image compression and image restoration [29, 37, 38]. Particularly, in [34-36], authors have shown the advantage of fractional order derivatives to achieve a good trade-off between image denoising and edge preservation. Also in [31-33] fractional differential mask has being proposed for contrast image enhancement. And in $[37,39,40]$ it has been demonstrated how using an edge detector based on fractional differentiation can improve the criterion of detection of thin artefacts in the image.

However any application of fractional calculation requires expressions for fractional derivatives and integrals. For example in [27], based on the definition of Riemann-Liouville and Caputo, expressions for fractional derivatives and integrals of some elementary functions are obtained.

Our purpose in this paper is to give some theoretical properties concerning the Caputo-Fabrizio fractional operators and apply these operators to some interesting elementary functions. The paper has been organized as follows, in Section 2 , 
we present basic definitions and formulas. In Section 3, we give theoretical properties of Caputo-Fabrizio fractional derivative. In section 4, we study the composition of Caputo-Fabrizio fractional operators. In section 5, we give examples on using Caputo-Fabrizio fractional integral. In Section 6, we give some examples on using Caputo-Fabrizio fractional derivative. A conclusion is considered in section 7.

\section{Preliminaries and notations}

Here, we introduce some definitions concerning the Caputo-Fabrizio fractional derivative and we give some formulas, which would be needed in our proofs later.

We denote by $C_{0}([a, b])$ the space of all continuous functions on $[a, b]$ with compact support.

Other interesting notation is

$$
C_{0}^{k}([a, b])=C^{k}([a, b]) \cap C_{0}([a, b])
$$

where $k \in \mathbb{Z}^{+} \cup\{\infty\}$.

Let $a, b, p \in \mathbb{R}$ with $1 \leq p \leq \infty$. The Sobolev space $W^{1, p}([a, b])$ is defined by

$$
W^{1, p}([a, b])=\left\{u \in L^{p}([a, b]) ; \exists g \in L^{p}([a, b]) \text { such that } \int_{a}^{b} u \cdot \varphi^{\prime}=-\int_{a}^{b} g \varphi, \quad \forall \varphi \in C_{0}^{\infty}([a, b])\right\} .
$$

Definition 1. Let $a, b, \alpha \in \mathbb{R}$ such that $0<\alpha<1$. The Caputo-Fabrizio fractional integral of order $\alpha$ of a function $u \in H^{1}([a, b])$ is a linear operator defined by

$$
I_{a t}^{\alpha} u(t)=(1-\alpha) u(t)+\alpha \int_{a}^{t} u(s) d s
$$

Definition 2. Let $a, b, \alpha \in \mathbb{R}$ such that $0<\alpha<1$. The Caputo-Fabrizio fractional derivative of order $\alpha$ of a function $u \in H^{1}([a, b])$ is a linear operator defined by

$$
D_{a t}^{\alpha} u(t)=\frac{1}{1-\alpha} \int_{a}^{t} e^{-\frac{\alpha}{1-\alpha}(t-s)} u^{\prime}(s) d s
$$

Definition 3. Let $\alpha>0$. The Riemann-Liouville fractional integral of order $\alpha$ of a function $u$ is defined by

$$
R_{a t}^{\alpha} u(t)=\frac{1}{\Gamma(\alpha)} \int_{a}^{t}(t-s)^{\alpha-1} u(s) d s .
$$

For more details, see [1-18].

Lemma 1. Let $n \in \mathbb{N}$ and $\alpha, a, b \in \mathbb{R}$. Then, for each $n$, the integral

$$
A_{n}(t)=n \int_{a}^{t} e^{\frac{-\alpha}{1-\alpha} t+\frac{\alpha+(1-\alpha) b}{1-\alpha} \tau}(\tau-a)^{n-1} d \tau
$$

can be written in the form

$$
A_{1}(t)=\frac{1-\alpha}{\alpha+(1-\alpha) b}\left(e^{b t}-e^{\frac{-\alpha}{1-\alpha} t+\frac{\alpha+(1-\alpha) b}{1-\alpha} a}\right)
$$


or

$$
\begin{aligned}
A_{n}(t) & =e^{b t} \sum_{i=1}^{n-1}(-1)^{i-1} \frac{n !}{(n-i) !}\left(\frac{1-\alpha}{\alpha+(1-\alpha) b}\right)^{i}(t-a)^{n-i} \\
& +(-1)^{n-1} n !\left(\frac{1-\alpha}{\alpha+(1-\alpha) b}\right)^{n}\left(e^{b t}-e^{\frac{-\alpha}{1-\alpha} t+\frac{\alpha+(1-\alpha) b}{1-\alpha} a}\right), \quad n>1
\end{aligned}
$$

Proof. We will use the principle of mathematical induction. Let $P(n)$ be

$$
P(n) \equiv A_{n}(t)
$$

For our base case, we need to show $P(1)$ is true, meaning that

$$
A_{1}(t)=\frac{1-\alpha}{\alpha+(1-\alpha) b}\left(e^{b t}-e^{\frac{-\alpha}{1-\alpha} t+\frac{\alpha+(1-\alpha) b}{1-\alpha} a}\right)
$$

This is trivial, since

$$
A_{1}(t)=\int_{a}^{t} e^{\frac{-\alpha}{1-\alpha} t+\frac{\alpha+(1-\alpha) b}{1-\alpha} \tau} d \tau
$$

For the inductive step, assume that for some $n, P(n)$ holds, so

$$
A_{n}(t)=n \int_{a}^{t} e^{\frac{-\alpha}{1-\alpha} t+\frac{\alpha+(1-\alpha) b}{1-\alpha} \tau}(\tau-a)^{n-1} d \tau .
$$

We need to show that $P(n+1)$ holds, meaning that

$$
A_{n+1}(t)=(n+1) \int_{a}^{t} e^{\frac{-\alpha}{1-\alpha} t+\frac{\alpha+(1-\alpha) b}{1-\alpha} \tau}(\tau-a)^{n} d \tau
$$

To see this, note that

$$
\begin{aligned}
A_{n+1}(t)= & (n+1) \int_{a}^{t} e^{\frac{-\alpha}{1-\alpha} t+\frac{\alpha+(1-\alpha) b}{1-\alpha} \tau}(\tau-a)^{n} d \tau \\
= & \frac{(1-\alpha)(n+1)}{\alpha+(1-\alpha) b}(t-a)^{n} e^{b t}-\frac{(1-\alpha)(n+1)}{\alpha+(1-\alpha) b} n \int_{a}^{t} e^{\frac{-\alpha}{1-\alpha} t+\frac{\alpha+(1-\alpha) b}{1-\alpha} \tau}(\tau-a)^{n-1} d \tau \\
= & \frac{(1-\alpha)(n+1)}{\alpha+(1-\alpha) b}(t-a)^{n} e^{b t}+e^{b t} \sum_{i=1}^{n-1}(-1)^{i} \frac{(n+1) n !}{(n-i) !}\left(\frac{1-\alpha}{\alpha+(1-\alpha) b}\right)^{i+1}(t-a)^{n-i} \\
& +(-1)^{n}(n+1) !\left(\frac{1-\alpha}{\alpha+(1-\alpha) b}\right)^{n+1}\left(e^{b t}-e^{\frac{-\alpha}{1-\alpha} t+\frac{\alpha+(1-\alpha) b}{1-\alpha} a}\right) \\
= & \frac{(1-\alpha)(n+1)}{\alpha+(1-\alpha) b}(t-a)^{n} e^{b t}+e^{b t} \sum_{i=2}^{n}(-1)^{i-1} \frac{(n+1) n !}{(n+1-i) !}\left\{\frac{1-\alpha}{\alpha+(1-\alpha) b}\right\}^{i}(t-a)^{n+1-i} \\
& +(-1)^{n}(n+1) !\left\{\frac{1-\alpha}{\alpha+(1-\alpha) b}\right\}^{n+1}\left(e^{b t}-e^{\frac{-\alpha}{1-\alpha} t+\frac{\alpha+1-\alpha) b}{1-\alpha} a}\right) \\
= & e^{b t} \sum_{i=1}^{n}(-1)^{i-1} \frac{(n+1) !}{(n+1-i) !}\left(\frac{1-\alpha}{\alpha+(1-\alpha) b}\right)^{i}(t-a)^{n+1-i} \\
& +(-1)^{n}(n+1) !\left(\frac{1-\alpha}{\alpha+(1-\alpha) b}\right)^{n+1}\left(e^{b t}-e^{\frac{-\alpha}{1-\alpha} t+\frac{\alpha+(1-\alpha) b}{1-\alpha} a}\right)
\end{aligned}
$$

Thus $P(n+1)$ holds when $P(n)$ is true, so $P(n)$ is true for all natural numbers $n$. 
NTMSCI 8, No. 1, 1-25 (2020) / www.ntmsci.com

SKA

5

Lemma 2. Let $n \in \mathbb{N}$ and $a, b, \alpha \in \mathbb{R}$ such that $\alpha \in(0,1)$. Then, for each $n$, the integral

$$
B_{n}(t)=\int_{a}^{t} e^{\frac{-\alpha}{1-\alpha} t+\frac{\alpha+(1-\alpha) b}{1-\alpha} \tau}(\tau-a)^{n} d \tau
$$

can be written in the form

$$
B_{1}(t)=(t-a) \frac{1-\alpha}{\alpha+(1-\alpha) b} e^{b t}-\left(\frac{1-\alpha}{\alpha+(1-\alpha) b}\right)^{2}\left(e^{b t}-e^{\frac{-\alpha}{1-\alpha} t+\frac{\alpha+(1-\alpha) b}{1-\alpha} a}\right)
$$

or

$$
\begin{aligned}
B_{n}(t)= & e^{b t} \sum_{i=1}^{n}(-1)^{i-1} \frac{n !}{(n+1-i) !}\left(\frac{1-\alpha}{\alpha+(1-\alpha) b}\right)^{i}(t-a)^{n-i} \\
& +(-1)^{n} n !\left(\frac{1-\alpha}{\alpha+(1-\alpha) b}\right)^{n+1}\left(e^{b t}-e^{\frac{-\alpha}{1-\alpha} t+\frac{\alpha+(1-\alpha) b}{1-\alpha} a}\right), \quad n>1
\end{aligned}
$$

Proof. Let $P(n)$ be

$$
P(n) \equiv B_{n}(t)
$$

We will show that $P(n)$ holds for all $n \in \mathbb{R}$ by induction. We note that

$$
\begin{aligned}
B_{1}(t) & =\int_{a}^{t} e^{\frac{-\alpha}{1-\alpha} t+\frac{\alpha+(1-\alpha) b}{1-\alpha} \tau}(\tau-a) d \tau \\
& =(t-a) \frac{1-\alpha}{\alpha+(1-\alpha) b} e^{b t}-\left(\frac{1-\alpha}{\alpha+(1-\alpha) b}\right)^{2}\left(e^{b t}-e^{\frac{-\alpha}{1-\alpha} t+\frac{\alpha+(1-\alpha) b}{1-\alpha} a}\right)
\end{aligned}
$$

Thus, $P(n)$ is true for $n=1$. Assume that $P(n)$ is true for some natural number $n>1$, ie.,

$$
B_{n}(t)=\int_{a}^{t} e^{\frac{-\alpha}{1-\alpha} t+\frac{\alpha+(1-\alpha) b}{1-\alpha} \tau}(\tau-a)^{n} d \tau
$$

We need to proof that $P(n)$ is true for $n+1$ whenever $P(n)$ is true for $n$. We have

Proof.

$$
\begin{aligned}
B_{n+1}(t)= & \int_{a}^{t} e^{\frac{-\alpha}{1-\alpha} t+\frac{\alpha+(1-\alpha) b}{1-\alpha} \tau}(\tau-a)^{n+1} d \tau \\
= & \frac{1-\alpha}{\alpha+(1-\alpha) b}(t-a)^{n+1} e^{b t}-(n+1) \frac{1-\alpha}{\alpha+(1-\alpha) b} \int_{a}^{t} e^{\frac{-\alpha}{1-\alpha} t+\frac{\alpha+(1-\alpha) b}{1-\alpha} \tau}(\tau-a)^{n} d \tau \\
= & \frac{(1-\alpha)(t-a)^{n+1} e^{b t}}{\alpha+(1-\alpha) b}-\frac{(n+1)(1-\alpha)}{\alpha+(1-\alpha) b} e^{b t} \sum_{i=1}^{n} \frac{(-1)^{i-1} n !}{(n+1-i) !}\left(\frac{1-\alpha}{\alpha+(1-\alpha) b}\right)^{i}(t-a)^{n+1-i} \\
& -(n+1) \frac{1-\alpha}{\alpha+(1-\alpha) b}(-1)^{n}(n !)\left(\frac{1-\alpha}{\alpha+(1-\alpha) b}\right)^{n+1}\left(e^{b t}-e^{\frac{-\alpha}{1-\alpha} t+\frac{\alpha+(1-\alpha) b}{1-\alpha} a}\right) \\
= & \frac{(1-\alpha)(t-a)^{n+1} e^{b t}}{\alpha+(1-\alpha) b}+e^{b t} \sum_{i=1}^{n}(-1)^{i} \frac{(n+1) n !}{(n+1-i) !}\left(\frac{1-\alpha}{\alpha+(1-\alpha) b}\right)^{i+1}(t-a)^{n+1-i} \\
& +\frac{1-\alpha}{\alpha+(1-\alpha) b}(-1)^{n+1}(n+1)(n !)\left(\frac{1-\alpha}{\alpha+(1-\alpha) b}\right)^{n+1}\left(e^{b t}-e^{\frac{-\alpha}{1-\alpha} t+\frac{\alpha+(1-\alpha) b}{1-\alpha} a}\right)
\end{aligned}
$$

(ㄷ) 2020 BISKA Bilisim Technology 


$$
\begin{aligned}
= & \frac{(1-\alpha)(t-a)^{n+1} e^{b t}}{\alpha+(1-\alpha) b}+e^{b t} \sum_{i=2}^{n+1}(-1)^{i-1} \frac{(n+1) !}{(n+1+1-i) !}\left(\frac{1-\alpha}{\alpha+(1-\alpha) b}\right)^{i}(t-a)^{n+1+1-i} \\
& +(-1)^{n+1}(n+1) !\left(\frac{1-\alpha}{\alpha+(1-\alpha) b}\right)^{n+1+1}\left(e^{b t}-e^{\frac{-\alpha}{1-\alpha} t+\frac{\alpha+(1-\alpha) b}{1-\alpha} a}\right) \\
= & e^{b t} \sum_{i=1}^{n+1}(-1)^{i-1} \frac{(n+1) !}{(n+2-i) !}\left(\frac{1-\alpha}{\alpha+(1-\alpha) b}\right)^{i}(t-a)^{n+2-i} \\
& +(-1)^{n+1}(n+1) !\left(\frac{1-\alpha}{\alpha+(1-\alpha) b}\right)^{n+2}\left(e^{b t}-e^{\frac{-\alpha}{1-\alpha} t+\frac{\alpha+(1-\alpha) b}{1-\alpha} a}\right)
\end{aligned}
$$

Thus, $P(n)$ is true for $n+1$ whenever $P(n)$ is true for $n$. Hence, by the principle of mathematical induction, $P(n)$ is true for all natural numbers $n$.

Proposition 1. For $1<n \in \mathbb{N}$, one has easily the following:

$$
\begin{gathered}
\int \frac{2 x+\frac{2 n}{2 n-1}}{\sqrt[n]{x^{2}+\frac{2 n}{2 n-1} x+\frac{2 n}{2 n-1}}} d x=\frac{n}{n-1} \sqrt[n]{\left(x^{2}+\frac{2 n}{2 n-1} x+\frac{2 n}{2 n-1}\right)^{n-1}} \\
\int\left(2 x+\frac{2 n}{2 n+1}\right) \sqrt[n]{x^{2}+\frac{2 n}{2 n+1} x+\frac{2 n}{2 n+1}} d x=\frac{n}{n+1}\left(x^{2}+\frac{2 n}{2 n+1} x+\frac{2 n}{2 n+1}\right)^{\frac{n+1}{n}}
\end{gathered}
$$

\section{Some properties cencerning the Caputo-Fabrizio fractional derivative}

Here, we give some theoretical properties concerning the Caputo-Fabrizio fractional derivative.

Theorem 1. Let be $u(t) \in C^{n+1}([a, b])$, then the equality

$$
\begin{aligned}
D_{a t}^{\alpha} u(t)= & (-1)^{n+2}\left(\frac{1-\alpha}{\alpha}\right)^{n} D_{a t}^{\alpha} u^{(n)}(t) \\
& +\sum_{k=1}^{n}(-1)^{k+1} \frac{(1-\alpha)^{k-1}}{\alpha^{k}}\left[u^{(k)}(t)-e^{\frac{-\alpha}{1-\alpha}(t-a)} u^{(k)}(a)\right]
\end{aligned}
$$

holds true.

Proof. Let $P(n)$ be

$$
P(n) \equiv D_{a t}^{\alpha} u(t)=(-1)^{n+2}\left(\frac{1-\alpha}{\alpha}\right)^{n} D_{a t}^{\alpha} u^{(n)}(t)+\sum_{k=1}^{n}(-1)^{k+1} \frac{(1-\alpha)^{k-1}}{\alpha^{k}}\left[u^{(k)}(t)-e^{\frac{-\alpha}{1-\alpha}(t-a)} u^{(k)}(a)\right]
$$

We will show, by induction, that $P(n)$ holds for all $n \in \mathbb{N}$. Integrating by parts, we note that

$$
\begin{aligned}
D_{a t}^{\alpha} u(t) & =\frac{1}{1-\alpha} \int_{a}^{t} e^{-\frac{\alpha}{1-\alpha}(t-s)} u^{\prime}(s) d s \\
& =\frac{1}{1-\alpha}\left[\frac{1-\alpha}{\alpha} \cdot u^{\prime}(t)-\frac{1-\alpha}{\alpha} e^{\frac{-\alpha}{1-\alpha}(t-a)} u^{\prime}(a)-\frac{1-\alpha}{\alpha} \int_{a}^{t} e^{-\frac{\alpha}{1-\alpha}(t-s)}\left(u^{\prime}(s)\right)^{\prime} d s\right] \\
& =\frac{1}{\alpha} \cdot u^{\prime}(t)-\frac{1}{\alpha} \cdot e^{\frac{-\alpha}{1-\alpha}(t-a)} u^{\prime}(a)-\frac{1-\alpha}{\alpha} \cdot \frac{1}{1-\alpha} \cdot \int_{a}^{t} e^{-\frac{\alpha}{1-\alpha}(t-s)}\left(u^{\prime}(s)\right)^{\prime} d s \\
& =-\frac{1-\alpha}{\alpha} \cdot D_{a t}^{\alpha} u^{\prime}(t)+\frac{1}{\alpha} \cdot u^{\prime}(t)-\frac{1}{\alpha} \cdot e^{\frac{-\alpha}{1-\alpha}(t-a)} u^{\prime}(a)
\end{aligned}
$$


Thus, $P(n)$ is true for $n=1$. Asume that $P(n)$ is true for some natural number $n$, i.e.,

$$
D_{a t}^{\alpha} u(t)=(-1)^{n+2}\left(\frac{1-\alpha}{\alpha}\right)^{n} D_{a t}^{\alpha} u^{(n)}(t)+\sum_{k=1}^{n}(-1)^{k+1} \frac{(1-\alpha)^{k-1}}{\alpha^{k}}\left[u^{(k)}(t)-e^{\frac{-\alpha}{1-\alpha}(t-a)} u^{(k)}(a)\right]
$$

We need to proof that $P(n)$ is true for $n+1$ whenever $P(n)$ is true for $n$. We have

$$
\begin{aligned}
D_{a t}^{\alpha} u(t)= & (-1)^{n+2}\left(\frac{1-\alpha}{\alpha}\right)^{n} D_{a t}^{\alpha} u^{(n)}(t)+\sum_{k=1}^{n}(-1)^{k+1} \frac{(1-\alpha)^{k-1}}{\alpha^{k}}\left[u^{(k)}(t)-e^{\frac{-\alpha}{1-\alpha}(t-a)} u^{(k)}(a)\right] \\
= & (-1)^{n+2}\left(\frac{1-\alpha}{\alpha}\right)^{n} \cdot \frac{1}{1-\alpha} \int_{a}^{t} e^{-\frac{\alpha}{1-\alpha}(t-s)} u^{(n+1)}(s) d s+\sum_{k=1}^{n}(-1)^{k+1} \frac{(1-\alpha)^{k-1}}{\alpha^{k}}\left[u^{(k)}(t)-e^{\frac{-\alpha}{1-\alpha}(t-a)} u^{(k)}(a)\right] \\
= & (-1)^{n+2}\left(\frac{1-\alpha}{\alpha}\right)^{n} \cdot \frac{1}{1-\alpha}\left[\frac{1-\alpha}{\alpha}\left(u^{(n+1)}(t)-e^{\frac{-\alpha}{1-\alpha}(t-a)} u^{(n+1)}(a)\right)-\frac{1-\alpha}{\alpha} \int_{a}^{t} e^{-\frac{\alpha}{1-\alpha}(t-s)}\left(u^{(n+1)}\right)^{\prime}(s) d s\right] \\
& +\sum_{k=1}^{n}(-1)^{k+1} \frac{(1-\alpha)^{k-1}}{\alpha^{k}}\left[u^{(k)}(t)-e^{\frac{-\alpha}{1-\alpha}(t-a)} u^{(k)}(a)\right] \\
= & (-1)^{n+1+2}\left(\frac{1-\alpha}{\alpha}\right)^{n} \cdot \frac{1}{1-\alpha} \int_{a}^{t} e^{-\frac{\alpha}{1-\alpha}(t-s)}\left(u^{(n+1)}\right)^{\prime}(s) d s+(-1)^{n+2} \frac{(1-\alpha)^{n}}{\alpha^{n+1} \cdot\left(u^{(n+1)}(t)-e^{\frac{-\alpha}{1-\alpha}(t-a)} u^{(n+1)}(a)\right)} \\
& +\sum_{k=1}^{n}(-1)^{k+1} \frac{(1-\alpha)^{k-1}}{\alpha^{k}}\left[u^{(k)}(t)-e^{\frac{-\alpha}{1-\alpha}(t-a)} u^{(k)}(a)\right] \\
= & (-1)^{n+1+2}\left(\frac{1-\alpha}{\alpha}\right)^{n+1} \cdot D_{a t}^{\alpha} u^{(n+1)}(t)+\sum_{k=1}^{n+1}(-1)^{k+1} \frac{(1-\alpha)^{k-1}}{\alpha^{k}}\left[u^{(k)}(t)-e^{\frac{-\alpha}{1-\alpha}(t-a)} u^{(k)}(a)\right] .
\end{aligned}
$$

Theorem 2. Let be $u \in C^{n+1}[a, b]$. Then the equality

$$
(1-\alpha) D_{a t}^{\alpha} u^{(n)}(t)+\alpha D_{a t}^{\alpha} u^{(n-1)}(t)=u^{(n)}(t)-e^{\frac{-\alpha}{1-\alpha}(t-a)} u^{(n)}(a)
$$

holds true for all $t \in[a, b]$

Proof. We insert $n$ and $n-1$ into the equality (19). This yields

$$
D_{a t}^{\alpha} u(t)=(-1)^{n+2}\left(\frac{1-\alpha}{\alpha}\right)^{n} D_{a t}^{\alpha} u^{(n)}(t)+\sum_{k=1}^{n}(-1)^{k+1} \frac{(1-\alpha)^{k-1}}{\alpha^{k}}\left[u^{(k)}(t)-e^{\frac{-\alpha}{1-\alpha}(t-a)} u^{(k)}(a)\right]
$$

and

$$
D_{a t}^{\alpha} u(t)=(-1)^{n+1}\left(\frac{1-\alpha}{\alpha}\right)^{n-1} D_{a t}^{\alpha} u^{(n-1)}(t)+\sum_{k=1}^{n-1}(-1)^{k+1} \frac{(1-\alpha)^{k-1}}{\alpha^{k}}\left[u^{(k)}(t)-e^{\frac{-\alpha}{1-\alpha}(t-a)} u^{(k)}(a)\right]
$$

respctively. Combining (25) with (26) we obtain (24). Theorem 2 is thus proved.

Theorem 3. Let be $n \in \mathbb{N}-\{0\}, a, b \in \mathbb{R}(a<b)$ and $u \in C^{n}([a, b])$. Then the equality

$$
\frac{d^{n}}{d t^{n}}\left(D_{a t}^{\alpha} u(t)\right)=\sum_{i=1}^{n}(-1)^{n-i} \frac{\alpha^{n-i}}{(1-\alpha)^{n+1-i}} u^{(i)}(t)+(-1)^{n}\left(\frac{\alpha}{1-\alpha}\right)^{n} D_{a t}^{\alpha} u(t)
$$

holds true. 
Proof. We will use the principle of mathematical induction. Let $P(n)$ be

$$
P(n) \equiv \frac{d^{n}}{d t^{n}}\left(D_{a t}^{\alpha} u(t)\right)=\sum_{i=1}^{n} \frac{(-\alpha)^{n-i}}{(1-\alpha)^{n+1-i}} u^{(i)}(t)+\left(\frac{-\alpha}{1-\alpha}\right)^{n} D_{a t}^{\alpha} u(t)
$$

For our base case, we need to show $P(1)$ is true, meaning that

$$
\frac{d}{d t}\left(D_{a t}^{\alpha} u(t)\right)=\frac{1}{1-\alpha} \sum_{i=1}^{1}(-1)^{1-i}\left(\frac{\alpha}{1-\alpha}\right)^{1-i} u^{(i)}+(-1)^{1}\left(\frac{\alpha}{1-\alpha}\right)^{1} D_{a t}^{\alpha} u(t)
$$

This is trivial, since

$$
\begin{aligned}
& \frac{d}{d t}\left(D_{a t}^{\alpha} u(t)\right)=\frac{d}{d t}\left[\frac{1}{1-\alpha} u(t)-\frac{1}{1-\alpha} e^{-\frac{\alpha}{1-\alpha}(t-a)} u(a)-\frac{\alpha}{(1-\alpha)^{2}} \int_{a}^{t} e^{-\frac{\alpha}{1-\alpha}(t-\tau)} u(\tau) d \tau\right] \\
& =\frac{1}{1-\alpha} u^{\prime}(t)+\frac{\alpha}{(1-\alpha)^{2}} e^{-\frac{\alpha}{1-\alpha}(t-a)} u(a)-\frac{\alpha}{(1-\alpha)^{2}} u(t)+\frac{\alpha^{2}}{(1-\alpha)^{3}} \int_{a}^{t} e^{-\frac{\alpha}{1-\alpha}(t-\tau)} u(\tau) d \tau \\
& =\frac{1}{1-\alpha} u^{\prime}(t)+\frac{\alpha}{(1-\alpha)^{2}} e^{-\frac{\alpha}{1-\alpha}(t-a)} u(a)-\frac{\alpha}{(1-\alpha)^{2}} u(t) \\
& -\frac{\alpha}{1-\alpha}\left[\frac{1}{1-\alpha} u(t)-\frac{1}{1-\alpha} e^{-\frac{\alpha}{1-\alpha}(t-a)} u(a)-\frac{\alpha}{(1-\alpha)^{2}}\right. \text {. } \\
& \left.\cdot \int_{a}^{t} e^{-\frac{\alpha}{1-\alpha}(t-\tau)} u(\tau) d \tau-\frac{1}{1-\alpha} u(t)+\frac{1}{1-\alpha} e^{-\frac{\alpha}{1-\alpha}(t-a)} u(a)\right] \\
& =\frac{1}{1-\alpha} u^{\prime}(t)+\frac{\alpha}{(1-\alpha)^{2}} e^{-\frac{\alpha}{1-\alpha}(t-a)} u(a)-\frac{\alpha}{(1-\alpha)^{2}} u(t) \\
& -\frac{\alpha}{1-\alpha}\left[D_{a t}^{\alpha} u(t)-\frac{1}{1-\alpha} u(t)+\frac{1}{1-\alpha} e^{-\frac{\alpha}{1-\alpha}(t-a)} u(a)\right] \\
& =\frac{1}{1-\alpha} \sum_{i=1}^{1}(-1)^{1-i}\left(\frac{\alpha}{1-\alpha}\right)^{1-i} u^{(i)}+(-1)^{1}\left(\frac{\alpha}{1-\alpha}\right)^{1} D_{a t}^{\alpha} u(t)
\end{aligned}
$$

For the inductive step, assume that for some $n, P(n)$ holds, so

$$
\frac{d^{n}}{d t^{n}}\left(D_{a t}^{\alpha} u(t)\right)=\frac{1}{1-\alpha} \sum_{i=1}^{n}(-1)^{n-i}\left(\frac{\alpha}{1-\alpha}\right)^{n-i} u^{(i)}+(-1)^{n}\left(\frac{\alpha}{1-\alpha}\right)^{n} D_{a t}^{\alpha} u(t)
$$

We need to show that $P(n+1)$ holds, meaning that

$$
\frac{d^{n+1}}{d t^{n+1}}\left(D_{a t}^{\alpha} u(t)\right)=\sum_{i=1}^{n+1} \frac{(-\alpha)^{n+1-i}}{(1-\alpha)^{n+2-i}} u^{(i)}(t)+\left(\frac{-\alpha}{1-\alpha}\right)^{n+1} D_{a t}^{\alpha} u(t)
$$


To see this, note that

$$
\begin{aligned}
\frac{d^{n+1}}{d t^{n+1}}\left(D_{a t}^{\alpha} u(t)\right) & =\frac{d^{n}}{d t^{n}}\left\{\frac{d}{d t} D_{a t}^{\alpha} u(t)\right\} \\
& =\frac{d^{n}}{d t^{n}}\left\{\frac{-\alpha}{1-\alpha} D_{a t}^{\alpha} u(t)+\frac{1}{1-\alpha} u^{\prime}(t)\right\} \\
& =\frac{-\alpha}{1-\alpha} \frac{d^{n}}{d t^{n}}\left(D_{a t}^{\alpha} u(t)\right)+\frac{1}{1-\alpha} u^{(n+1)}(t) \\
& =\frac{-\alpha}{1-\alpha}\left[\frac{1}{1-\alpha} \sum_{i=1}^{n}(-1)^{k-i}\left(\frac{\alpha}{1-\alpha}\right)^{n-i} u^{(i)}+(-1)^{n}\left(\frac{\alpha}{1-\alpha}\right)^{n} D_{a t}^{\alpha} u(t)\right]+\frac{1}{1-\alpha} u^{(n+1)}(t) \\
& =\left[\frac{1}{1-\alpha} \sum_{i=1}^{n}(-1)^{n+1-i}\left(\frac{\alpha}{1-\alpha}\right)^{n+1-i} u^{(i)}+(-1)^{n+1}\left(\frac{\alpha}{1-\alpha}\right)^{n+1} D_{a t}^{\alpha} u(t)\right]+\frac{1}{1-\alpha} u^{(n+1)}(t) \\
& =\frac{1}{1-\alpha} \sum_{i=1}^{n+1}(-1)^{n+1-i}\left(\frac{\alpha}{1-\alpha}\right)^{n+1-i} u^{(i)}+(-1)^{n+1}\left(\frac{\alpha}{1-\alpha}\right)^{n+1} D_{a t}^{\alpha} u(t)
\end{aligned}
$$

thus $P(n+1)$ holds when $P(n)$ is true, so $P(n)$ is true for all natural numbers $n$.

Corollary 1. Let be $a, b \in \mathbb{R}(a<b)$ and $u \in C^{1}([a, b])$. Then the equality

$$
\int_{a}^{b}\left(D_{a t}^{\alpha} u(t)\right) d t=\frac{1}{\alpha} \cdot(u(b)-u(a))-\frac{1-\alpha}{\alpha} D_{a b}^{\alpha} u(b)
$$

holds true

Proof. Applying (27) for $n=1$, we obtain

$$
\frac{d}{d t}\left(D_{a t}^{\alpha} u(t)\right)=\frac{1}{1-\alpha} u^{\prime}(t)-\frac{\alpha}{1-\alpha} D_{a t}^{\alpha} u(t)
$$

Then integrating (34) with respect to $t$ over $(a, b)$ and considering that $D_{a a}^{\alpha} u(a)=0$, we obtain (33).

Theorem 4. Let be $u \in C^{1}[a, b] \cap H^{1}[a, b]$. Then $D_{a t}^{\alpha} u(t) \in H^{1}[a, b]$.

Proof. We need to proof that $D_{a t}^{\alpha} u(t), \frac{d}{d t}\left(D_{a t}^{\alpha} u(t)\right) \in L^{2}[a, b]$. On the one hand, we note that

$$
D_{a t}^{\alpha} u(t)=\frac{1}{1-\alpha} \int_{a}^{t} e^{-\frac{\alpha}{1-\alpha}(t-s)} u^{\prime}(s) d s=\frac{1}{1-\alpha}\left(u(t)-u(a) e^{-\frac{\alpha}{1-\alpha}(t-a)}-\frac{\alpha}{1-\alpha} \int_{a}^{t} e^{-\frac{\alpha}{1-\alpha}(t-s)} u(s) d s\right) .
$$

Then we can write

$$
\begin{aligned}
\left|D_{a t}^{\alpha} u(t)\right|^{2} \leq & \left(\frac{1}{1-\alpha}\right)^{2}\left[|u(t)|^{2}+\left(|u(a)|+\frac{\alpha}{1-\alpha} \cdot(b-a)^{1 / 2} \cdot\|u(t)\|_{L^{2}[a, b]}\right)^{2}\right. \\
& \left.+2\left(|u(a)|+\frac{\alpha}{1-\alpha} \cdot(b-a)^{1 / 2} \cdot\|u(t)\|_{L^{2}[a, b]}\right) \cdot|u(t)|\right]
\end{aligned}
$$

And consequently,

$$
\begin{aligned}
\left\|D_{a t}^{\alpha} u(t)\right\|_{L^{2}[a, b]}^{2} \leq & \left(\frac{1}{1-\alpha}\right)^{2}\left[\|u(t)\|_{L^{2}[a, b]}^{2}+\left(|u(a)|+\frac{\alpha}{1-\alpha} \cdot(b-a)^{1 / 2} \cdot\|u(t)\|_{L^{2}[a, b]}\right)^{2}(b-a)\right. \\
& \left.+2\left(|u(a)|+\frac{\alpha}{1-\alpha} \cdot(b-a)^{1 / 2} \cdot\|u(t)\|_{L^{2}[a, b]}\right) \cdot(b-a)^{1 / 2} \cdot\|u\|_{L^{2}[a, b]}\right]<\infty .
\end{aligned}
$$


On the other hand, applying Theorem 3, we obtain

$$
\frac{d}{d t}\left(D_{a t}^{\alpha} u(t)\right)=\frac{1}{1-\alpha} u^{\prime}(t)-\frac{\alpha}{1-\alpha} D_{a t}^{\alpha} u(t)
$$

As $u^{\prime}, D_{a t}^{\alpha} u(t) \in L^{2}[a, b]$, we conclude that $\frac{d}{d t}\left(D_{a t}^{\alpha} u(t)\right) \in L^{2}[a, b]$.

\section{Composition of Fractional Operators}

In this section, we give some theoretical properties concerning the composition of Caputo-Fabrizio fractional operators.

Theorem 5. Let be a, $\alpha, \beta \in \mathbb{R}$ such that $0<\alpha, \beta<1(\alpha \neq \beta)$. Then the inequality

$$
D_{a t}^{\alpha}\left(D_{a t}^{\beta} u(t)\right)=\frac{1}{\beta-\alpha}\left(\beta \cdot D_{a t}^{\beta} u(t)-\alpha \cdot D_{a t}^{\alpha} u(t)\right)
$$

holds true.

Proof. On the one side, from the definition of Caputo-Fabrizio derivative, we deduce that

$$
\begin{aligned}
D_{a t}^{\alpha}\left(D_{a t}^{\beta} u(t)\right) & =\frac{1}{1-\alpha} \int_{a}^{t} e^{-\frac{\alpha}{1-\alpha}(t-\tau)}\left(D_{a \tau}^{\beta} u(\tau)\right)^{\prime} d \tau \\
& =\frac{1}{1-\alpha} \frac{1}{1-\beta} \int_{a}^{t} e^{-\frac{\alpha}{1-\alpha}(t-\tau)}\left[u^{\prime}(\tau)-\int_{a}^{\tau} \frac{\beta}{1-\beta} e^{-\frac{\beta}{1-\beta}(\tau-s)} u^{\prime}(s) d s\right] d \tau \\
& =\frac{1}{1-\alpha} \frac{-\beta}{1-\beta} \int_{a}^{t} e^{-\frac{\alpha}{1-\alpha}(t-\tau)} D_{a \tau}^{\beta} u(\tau) d \tau+\frac{1}{1-\beta} D_{a t}^{\alpha} u(t)
\end{aligned}
$$

which is equivalent to

$$
\int_{a}^{t} e^{-\frac{\alpha}{1-\alpha}(t-\tau)} D_{a \tau}^{\beta} u(\tau) d \tau=-\frac{(1-\alpha)(1-\beta)}{\beta} D_{a t}^{\alpha}\left(D_{a t}^{\beta} u(t)\right)+\frac{1-\alpha}{\beta} D_{a t}^{\alpha} u(t) .
$$

On the other side, integrating by parts and considering that

$$
D_{a a}^{\beta} u(a)=0,
$$

we obtain

$$
D_{a t}^{\alpha}\left(D_{a t}^{\beta} u(t)\right)=\frac{1}{1-\alpha} \int_{a}^{t} e^{-\frac{\alpha}{1-\alpha}(t-\tau)}\left(D_{a \tau}^{\beta} u(\tau)\right)^{\prime} d \tau,=\frac{1}{1-\alpha} D_{a t}^{\beta} u(t)-\frac{\alpha}{(1-\alpha)^{2}} \int_{a}^{t} e^{-\frac{\alpha}{1-\alpha}(t-\tau)} D_{a \tau}^{\beta} u(\tau) d \tau
$$

which is equivalent to

$$
\int_{a}^{t} e^{-\frac{\alpha}{1-\alpha}(t-\tau)} D_{a \tau}^{\beta} u(\tau) d \tau=\frac{1-\alpha}{\alpha} D_{a t}^{\beta} u(t)-\frac{(1-\alpha)^{2}}{\alpha} D_{a t}^{\alpha}\left(D_{a t}^{\beta} u(t)\right)
$$

Combining (38) with (39), we obtain (35).

Theorem 6. Let be $a, \alpha \in \mathbb{R}$ such that $0<\alpha<1$. Then the equality

$$
D_{a t}^{\alpha}\left(I_{a t}^{\alpha} u(t)\right)=u(t)-e^{-\frac{\alpha}{1-\alpha}(t-a)} \cdot u(a),
$$

holds true. 
Proof. Applying definition of Caputo-Fabrizio derivative, we obtain

$$
\begin{aligned}
D_{a t}^{\alpha}\left(I_{a t}^{\alpha} u(t)\right) & =\frac{1}{1-\alpha} \cdot \int_{a}^{t} e^{-\frac{\alpha}{1-\alpha}(t-\tau)}\left[I_{a \tau}^{\alpha} u(\tau)\right]^{\prime} d \tau \\
& =\frac{1}{1-\alpha} \cdot \int_{a}^{t} e^{-\frac{\alpha}{1-\alpha}(t-\tau)}\left[(1-\alpha) u(\tau)+\alpha \int_{a}^{\tau} u(s) d s\right]^{\prime} d \tau \\
& =\int_{a}^{t} e^{-\frac{\alpha}{1-\alpha}(t-\tau)} u^{\prime}(\tau) d \tau+\frac{\alpha}{1-\alpha} \int_{a}^{t} e^{-\frac{\alpha}{1-\alpha}(t-\tau)} u(\tau) d \tau \\
& =\int_{a}^{t} e^{-\frac{\alpha}{1-\alpha}(t-\tau)} u^{\prime}(\tau) d \tau+u(t)-e^{-\frac{\alpha}{1-\alpha}(t-a)} u(a)-\int_{a}^{t} e^{-\frac{\alpha}{1-\alpha}(t-\tau)} u^{\prime}(\tau) d \tau \\
& =u(t)-e^{-\frac{\alpha}{1-\alpha}(t-a)} u(a),
\end{aligned}
$$

as required.

Theorem 7. Let $a, \alpha \in \mathbb{R}$ such that $0<\alpha<1$. Then the equality

$$
I_{a t}^{\alpha}\left(I_{a t}^{\alpha} u(t)\right)=u(t)-u(a)
$$

holds true.

Proof. On the one side, using definition of Caputo-Fabrizio integral, we obtain

$$
I_{a t}^{\alpha}\left(D_{a t}^{\alpha} u(t)\right)=(1-\alpha) D_{a t}^{\alpha} u(t)+\alpha \int_{a}^{t} D_{a s}^{\alpha} u(s) d s
$$

On the other side, applying Theorem 3, we obtain

$$
\frac{d}{d s}\left[D_{a s}^{\alpha} u(s)\right]=-\frac{\alpha}{1-\alpha} D_{a s}^{\alpha} u(s)+\frac{1}{1-\alpha} u^{\prime}(s),
$$

Integrating (43) respect to $s$ over $(a, t)$ to $t$ and considering that $D_{a a}^{\alpha} f(a)=0$, we obtain

$$
\int_{a}^{t} D_{a s}^{\alpha} u(s) d s=\frac{1}{\alpha}[u(t)-u(a)]-\frac{1-\alpha}{\alpha} \cdot D_{a t}^{\alpha} u(t) .
$$

Inserting the right hand side of (44) into (42), we obtain (41) and thus the proof is completed.

Theorem 8. Let $0<\alpha<1, a \in \mathbb{R}$. Then the equality

$$
I_{a t}^{\alpha}\left(R_{a t}^{\alpha} u(t)\right)=(1-\alpha) R_{a t}^{\alpha} u(t)+\frac{\Gamma(\alpha+1)}{\Gamma(\alpha)} R_{a t}^{\alpha+1} u(t),
$$

holds true. 
Proof. Using the definition of the Caputo-Fabrizio integral and the Riemann-Liouville integral, we obtain

$$
\begin{aligned}
I_{a t}^{\alpha}\left(R_{a t}^{\alpha} u(t)\right) & =(1-\alpha) R_{a t}^{\alpha} u(t)+\alpha \int_{a}^{t} R_{a \tau}^{\alpha} u(\tau) d \tau \\
& =(1-\alpha) R_{a t}^{\alpha} u(t)+\alpha \int_{a}^{t}\left[\frac{1}{\Gamma(\alpha)} \int_{a}^{\tau}(\tau-\xi)^{\alpha-1} u(\xi) d \xi\right] d \tau \\
& =(1-\alpha) R_{a t}^{\alpha} u(t)+\frac{\alpha}{\Gamma(\alpha)} \int_{a}^{t} u(\xi) d \xi \int_{\xi}^{t}(\tau-\xi)^{\alpha-1} d \tau \\
& =(1-\alpha) R_{a t}^{\alpha} u(t)+\frac{\alpha}{\Gamma(\alpha)} \int_{a}^{t} \frac{1}{\alpha}(t-\xi)^{\alpha} u(\xi) d \xi \\
& =(1-\alpha) R_{a t}^{\alpha} u(t)+\frac{1}{\Gamma(\alpha)} \int_{a}^{t}(t-\xi)^{(\alpha+1)-1} u(\xi) d \xi \\
& =(1-\alpha) R_{a t}^{\alpha} u(t)+\frac{\Gamma(\alpha+1)}{\Gamma(\alpha) \Gamma(\alpha+1)} \int_{a}^{t}(t-\xi)^{(\alpha+1)-1} u(\xi) d \xi \\
& =(1-\alpha) R_{a t}^{\alpha} u(t)+\frac{\Gamma(\alpha+1)}{\Gamma(\alpha)} R_{a t}^{\alpha+1} u(t)
\end{aligned}
$$

where we have used

$$
\int_{\xi}^{t}(\tau-\xi)^{\alpha-1} d \tau=\frac{(\tau-\xi)^{\alpha}}{\alpha}
$$

\section{Fractional Integral of Caputo-Fabrizio for some elementary functions}

In this section we give explicit formulas for fractional integral of Caputo-Fabrizio of the following elementary functions

$$
\begin{aligned}
& \frac{x}{\sqrt[n]{x^{2}+a x+b}}, \\
& x \sqrt[n]{x^{2}+a x+b}, \\
& \sqrt[n]{x^{2}+a x+b}, \\
& \frac{1}{\sqrt[n]{x^{2}+a x+b}}, \\
& x^{2} \sqrt[n]{x^{2}+a x+b}, \\
& \frac{x^{2}}{\sqrt[n]{x^{2}+a x+b}}
\end{aligned}
$$

where $a, b \in \mathbb{R}$, such that both are not zero simultaneously, $1<n \in \mathbb{N}$ and $C$ will represent a generic constant. These formulas will be formulated as propositions. Further it is important to highlight that to obtain these formulas, an intensive auxiliary calculation work was necessary, which will be presented in the form of lemmas.

Proposition 2.Let be $u(x)=x \cdot \sqrt[n]{x^{2}+a x+b}$. Then the Caputo-Fabrizio fractional integral of $u(x)$ is given by

$$
I_{a x}^{\alpha} u(x)=(1-\alpha) \cdot u(x)+\alpha \cdot p_{4}(x)+C,
$$

where $p_{4}(x)$ is given as (71).

To proof Proposition 2, we need the following lemmas

Lemma 3.The equality

$$
\int x^{2} \sqrt[n]{x^{2}+\frac{2 n}{2 n+1} x+\frac{2 n}{2 n+1}} d x=\frac{n}{(3 n+2)}\left(x^{2}+\frac{2 n}{2 n+1} x+\frac{2 n}{2 n+1}\right)^{\frac{n+1}{n}}\left(x-\frac{n}{n+1}\right)+C
$$


holds true

Proof. It is easy to see, after integrating by parts, that

$$
\begin{aligned}
\int\left(x^{2}+\frac{2 n}{2 n+1} x+\frac{2 n}{2 n+1}\right) \sqrt[n]{x^{2}+\frac{2 n}{2 n+1} x+\frac{2 n}{2 n+1}} d x= & x\left(x^{2}+\frac{2 n}{2 n+1} x+\frac{2 n}{2 n+1}\right)^{\frac{n+1}{n}} \\
& -\frac{n+1}{n} \int\left(2 x^{2}+\frac{2 n}{2 n+1} x\right) \sqrt[n]{x^{2}+\frac{2 n}{2 n+1} x+\frac{2 n}{2 n+1} d x}
\end{aligned}
$$

which is equivalent to

$$
\int x^{2} \sqrt[n]{x^{2}+\frac{2 n}{2 n+1} x+\frac{2 n}{2 n+1}} d x=\frac{n}{3 n+2}\left[x\left(x^{2}+\frac{2 n}{2 n+1} x+\frac{2 n}{2 n+1}\right)^{\frac{n+1}{n}}-\int\left(2 x+\frac{2 n}{2 n+1}\right) \sqrt[n]{x^{2}+\frac{2 n}{2 n+1} x+\frac{2 n}{2 n+1}} d x\right]
$$

Following equalities (18) and (54), we conclude the proof.

Lemma 4. Let $a, b \in \mathbb{R}$ such that both are not equal to zero. If

$$
\alpha=\frac{\beta^{2}+a \beta+b}{2 \beta+a}
$$

and

$$
\beta=\frac{-a}{2}+\frac{1}{2} \sqrt{a^{2}-2 \frac{(2 n+1) a^{2}-2 n b}{3 n+2}}
$$

then we have

$$
\frac{1}{\alpha}(2 \beta+a)=\frac{1}{\alpha^{2}}\left(\beta^{2}+a \beta+b\right)=\frac{2 n}{2 n+1}
$$

Proof. We shall prove the first equality of (57), i.e

$$
\frac{1}{\alpha}(2 \beta+a)=\frac{1}{\alpha^{2}}\left(\beta^{2}+a \beta+b\right)
$$

Replacing (55) into both sides of (58), we obtain the same result, which is

$$
\frac{(2 \beta+a)^{2}}{\beta^{2}+a \beta+b}
$$

To verify the second equality of (57), we take (56) into (59), and we have

$$
\begin{aligned}
(2 \beta+a)^{2} & =\frac{n\left(4 b-a^{2}\right)}{3 n+2} \\
\beta^{2}+a \beta+b & =\frac{\left(4 b-a^{2}\right)(1+2 n)}{2(3 n+2)}
\end{aligned}
$$

Then, combining (60)-(61) with (59), we deduce that

$$
\frac{(2 \beta+a)^{2}}{\beta^{2}+a \beta+b}=\frac{n\left(4 b-a^{2}\right)}{3 n+2} \frac{2(3 n+2)}{\left(4 b-a^{2}\right)(1+2 n)}=\frac{2 n}{2 n+1}
$$

as required. 


\section{Lemma 5. The equality}

$$
\int(x-\beta)^{2} \sqrt[n]{x^{2}+a x+b} d x=p_{3}(x)+C
$$

holds true, where

$$
p_{3}(x)=\frac{n \alpha^{\frac{3 n+2}{n}}}{3 n+2}\left[\left(\frac{x-\beta}{\alpha}\right)^{2}+\frac{2 n}{2 n+1}\left(\frac{x-\beta}{\alpha}\right)+\frac{2 n}{2 n+1}\right]^{\frac{n+1}{n}}\left(\frac{x-\beta}{\alpha}-\frac{n}{n+1}\right)
$$

$\alpha$ and $\beta$ are given by (55) and (56), respectively.

Proof. Combining the change of variable

$$
x=\alpha t+\beta
$$

with Lemma 4, we deduce that

$$
\begin{aligned}
\int(x-\beta)^{2} \sqrt[n]{x^{2}+a x+b} d x & =\alpha \int(\alpha t)^{2} \sqrt[n]{(\alpha t+\beta)^{2}+a(\alpha t+\beta)+b} d t \\
& =\alpha \sqrt[3]{\alpha^{2}} \int t^{2} \sqrt[n]{t^{2}+\frac{1}{\alpha}(2 \beta+a) t+\frac{1}{\alpha^{2}}}\left(\beta^{2}+a \beta+b\right) d t \\
& =\alpha^{3} \sqrt[n]{\alpha^{2}} \int t^{2} \sqrt[n]{t^{2}+\frac{2 n}{2 n+1} t+\frac{2 n}{2 n+1}} d t
\end{aligned}
$$

Consequently, we have the assertion (62) with the aid of Lemma 3.

Lemma 6. The equality

$$
\begin{aligned}
\int\left(x+\frac{n b-\beta^{2}(3 n+2)}{2 \beta(3 n+2)+a(2 n+1)}\right) \sqrt[n]{x^{2}+a x+b} d x= & \frac{n}{2 \beta(3 n+2)+a(2 n+1)}\left[x\left(x^{2}+a x+b\right)^{\frac{n+1}{n}}-\alpha^{\frac{3 n+2}{n}}\left(\left(\frac{x-\beta}{\alpha}\right)^{2}\right.\right. \\
& \left.\left.+\frac{2 n}{2 n+1}\left(\frac{x-\beta}{\alpha}\right)+\frac{2 n}{2 n+1}\right)^{\frac{n+1}{n}}\left(\frac{x-\beta}{\alpha}-\frac{n}{n+1}\right)\right]+C
\end{aligned}
$$

holds true, where $\alpha$ and $\beta$ are given by (55) and (56), respectively.

Proof. It is easy to see, after integrating by parts, that

$$
\int \sqrt[n]{\left(x^{2}+a x+b\right)^{n+1}} d x=x\left(x^{2}+a x+b\right)^{\frac{n+1}{n}}-\frac{n+1}{n} \int\left(2 x^{2}+a x\right) \sqrt[n]{x^{2}+a x+b} d x .
$$

which is equivalent to

$$
\begin{aligned}
& \int\left(x+\frac{n b-(3 n+2) \beta^{2}}{2 \beta(3 n+2)+a(2 n+1)}\right) \sqrt[n]{x^{2}+a x+b} d x \\
& =\frac{n x\left(x^{2}+a x+b\right)^{\frac{n+1}{n}}}{2 \beta(3 n+2)+a(2 n+1)}-\frac{3 n+2}{2 \beta(3 n+2)+a(2 n+1)} \int(x-\beta)^{2} \sqrt[n]{x^{2}+a x+b}
\end{aligned}
$$

By (62) and (67), we obtain (66).

Lemma 7. The following statements are equivalents

(a) $\theta=\frac{n \cdot\left(\theta^{2}+a \theta+b\right)-\beta^{2}(3 n+2)}{2 \beta(3 n+2)+(2 \theta+a)(2 n+1)}$,
(b) $\theta=\frac{-[(n+1) a+(6 n+4) \beta] \pm \sqrt{\Delta_{1}}}{2(3 n+2)}$,

where $\beta$ is given by (56) and $\Delta_{1}$, by

$$
\Delta_{1}=(n+1)^{2} a^{2}+2(n+1)(6 n+4) a \beta+4 n b(3 n+2)
$$


Proof.

$$
\begin{aligned}
\theta & =\frac{n \cdot\left(\theta^{2}+a \theta+b\right)-\beta^{2}(3 n+2)}{2 \beta(3 n+2)+(2 \theta+a)(2 n+1)} \\
& \Leftrightarrow(6 n+4) \beta \theta+(4 n+2) \theta^{2}+a(2 n+1) \theta=n\left(\theta^{2}+a \theta+b\right)-\beta^{2}(3 n+2) \\
& \Leftrightarrow(3 n+2) \theta^{2}+[(n+1) a+(6 n+4) \beta] \theta+\left[\beta^{2}(3 n+2)-n b\right]=0 \\
& \Leftrightarrow \theta=\frac{-[(n+1) a+(6 n+4) \beta] \pm \sqrt{\Delta_{1}}}{2(3 n+2)}
\end{aligned}
$$

as required.

Lemma 8. The equality

$$
\int x \sqrt[n]{x^{2}+a x+b} d x=p_{4}(x)+C
$$

holds true, where

$$
\begin{aligned}
p_{4}(x)= & \frac{n}{2 \beta(3 n+2)+(2 \theta+a)(2 n+1)}(x-\theta)\left[(x-\theta)^{2}+(2 \theta+a)(x-\theta)\right. \\
& \left.+\left(\theta^{2}+a \theta+b\right)\right]^{\frac{n+1}{n}}-\frac{n \alpha^{\frac{3 n+2}{n}}}{[2 \beta(3 n+2)+(2 \theta+a)(2 n+1)]}\left[\left(\frac{x-\theta-\beta}{\alpha}\right)^{2}\right. \\
& \left.+\frac{2 n}{2 n+1}\left(\frac{x-\theta-\beta}{\alpha}\right)+\frac{2 n}{2 n+1}\right]^{\frac{n+1}{n}}\left(\frac{x-\theta-\beta}{\alpha}-\frac{n}{n+1}\right)
\end{aligned}
$$

$\alpha, \beta$ and $\theta$ are given by (55), (56) and (69), respectively.

Proof. From Lemma 7, we deduce that

$$
\theta=\frac{n \cdot\left(\theta^{2}+a \theta+b\right)-\beta^{2}(3 n+2)}{2 \beta(3 n+2)+(2 \theta+a)(2 n+1)}
$$

which is equivalent to

$$
t+\theta=t+\frac{n \cdot\left(\theta^{2}+a \theta+b\right)-\beta^{2}(3 n+2)}{2 \beta(3 n+2)+(2 \theta+a)(2 n+1)}
$$

In terms of the change of variable

$$
x=t+\theta
$$

and (72), we get

$$
\begin{aligned}
\int x \sqrt[n]{x^{2}+a x+b} d x=\int(t+\theta) \sqrt[n]{t^{2}+(2 \theta+a) t+\left(\theta^{2}+a \theta+b\right)} d t \\
\int\left(t+\frac{n \cdot\left(\theta^{2}+a \theta+b\right)-\beta^{2}(3 n+2)}{2 \beta(3 n+2)+(2 \theta+a)(2 n+1)}\right) \sqrt[n]{t^{2}+(2 \theta+a) t+\left(\theta^{2}+a \theta+b\right)} d t
\end{aligned}
$$

which, in conjunction with Lemma 6, we conclude the proof.

We now give the proof of Proposition 2

Proof. Combining definition 1 with the lemma 8, we obtain 52 .

Proposition 3.Let be $u(x)=\frac{x}{\sqrt[n]{x^{2}+a x+b}}$. Then the Caputo-Fabrizio fractional integral of $u(x)$ is given by

$$
I_{a x}^{\alpha} u(x)=(1-\alpha) \cdot u(x)+\alpha \cdot p_{2}(x)+C,
$$


where $p_{2}(x)$ is given as (99).

To proof Proposition 3, we need the following lemmas

Lemma 9. The equality

$$
\int \frac{x^{2}}{\sqrt[n]{x^{2}+\frac{2 n}{2 n-1} x+\frac{2 n}{2 n-1}}} d x=\frac{n}{3 n-2}\left(x-\frac{n}{n-1}\right)\left(x^{2}+\frac{2 n}{2 n-1} x+\frac{2 n}{2 n-1}\right)^{\frac{n-1}{n}}+C
$$

holds true

Proof. It is easy to see, after integrating by parts, that

$$
\int \sqrt[n]{\left(x^{2}+\frac{2 n}{2 n-1} x+\frac{2 n}{2 n-1}\right)^{n-1}} d x=x\left(x^{2}+\frac{2 n}{2 n-1} x+\frac{2 n}{2 n-1}\right)^{\frac{n-1}{n}}-\frac{n-1}{n} \int \frac{2 x^{2}+\frac{2 n}{2 n-1} x}{\sqrt[n]{x^{2}+\frac{2 n}{2 n-1} x+\frac{2 n}{2 n-1}}} d x
$$

which is equivalent to

$$
\int \frac{x^{2}}{\sqrt[n]{x^{2}+\frac{2 n}{2 n-1} x+\frac{2 n}{2 n-1}}} d x=\frac{n}{3 n-2} x\left(x^{2}+\frac{2 n}{2 n-1} x+\frac{2 n}{2 n-1}\right)^{\frac{n-1}{n}}-\frac{n}{3 n-2} \int \frac{2 x+\frac{2 n}{2 n-1}}{\sqrt[n]{x^{2}+\frac{2 n}{2 n-1} x+\frac{2 n}{2 n-1}}} d x
$$

Combining (17) with (77), equality (75) follows.

Lemma 10. Let $a, b \in \mathbb{R}$ such that both are not equal to zero. If

$$
\alpha=\frac{\beta^{2}+a \beta+b}{2 \beta+a}
$$

and

$$
\beta=\frac{-a}{2}+\frac{1}{2} \sqrt{a^{2}-2 \frac{(2 n-1) a^{2}-2 n b}{3 n-2}}
$$

then equalities

$$
\frac{1}{\alpha}(2 \beta+a)=\frac{1}{\alpha^{2}}\left(\beta^{2}+a \beta+b\right)=\frac{2 n}{2 n-1}
$$

hold

Proof. We shall prove the first equality of (80), i.e

$$
\frac{1}{\alpha}(2 \beta+a)=\frac{1}{\alpha^{2}}\left(\beta^{2}+a \beta+b\right)
$$

Replacing (78) into both sides of (81), we obtain the same result, which is

$$
\frac{(2 \beta+a)^{2}}{\beta^{2}+a \beta+b}
$$

To verify the second equality of (80), we take (79) into (82), and we have

$$
\begin{aligned}
(2 \beta+a)^{2} & =\frac{n\left(4 b-a^{2}\right)}{3 n-2} \\
\beta^{2}+a \beta+b & =\frac{\left(4 b-a^{2}\right)(2 n-1)}{2(3 n-2)}
\end{aligned}
$$


Then, in terms of (83)-(84) and (82), we obtain

$$
\frac{(2 \beta+a)^{2}}{\beta^{2}+a \beta+b}=\frac{n\left(4 b-a^{2}\right)}{3 n-2} \frac{2(3 n-2)}{\left(4 b-a^{2}\right)(2 n-1)}=\frac{2 n}{2 n-1},
$$

as required.

Lemma 11. The equality

$$
\int \frac{(x-\beta)^{2}}{\sqrt[n]{x^{2}+a x+b}} d x=p_{1}(x)+C
$$

holds true, where

$$
p_{1}(x)=\frac{n \alpha^{\frac{3 n-2}{n}}}{3 n-2}\left[\left(\frac{x-\beta}{\alpha}\right)^{2}+\frac{2 n}{2 n-1}\left(\frac{x-\beta}{\alpha}\right)+\frac{2 n}{2 n-1}\right]^{\frac{n-1}{n}}\left(\frac{x-\beta}{\alpha}-\frac{n}{n-1}\right)
$$

$\alpha$ and $\beta$ are given by (78) and (79), respectively.

Proof. By using the change of variable

$$
x=\alpha t+\beta
$$

and Lemma 10, we obtain

$$
\begin{aligned}
\int \frac{(x-\beta)^{2}}{\sqrt[n]{x^{2}+a x+b}} d x & =\alpha \int \frac{(\alpha t)^{2}}{\sqrt[n]{(\alpha t+\beta)^{2}+a(\alpha t+\beta)+b}} d t \\
& =\frac{\alpha^{3}}{\sqrt[n]{\alpha^{2}}} \int \frac{t^{2}}{\sqrt[n]{t^{2}+\frac{1}{\alpha}(2 \beta+a) t+\frac{1}{\alpha^{2}}\left(\beta^{2}+a \beta+b\right)}} d t \\
& =\alpha^{\frac{3 n-2}{n}} \int \frac{t^{2}}{\sqrt[n]{t^{2}+\frac{2 n}{2 n-1} t+\frac{2 n}{2 n-1}}} d t
\end{aligned}
$$

By (75) and (88) as well as (89), we conclude the proof.

Lemma 12. The equality

$$
\begin{aligned}
\int \frac{x+\frac{n b-\beta^{2}(3 n-2)}{2 \beta(3 n-2)+a(2 n-1)}}{\sqrt[n]{x^{2}+a x+b}} d x= & \frac{n}{2 \beta(3 n-2)+a(2 n-1)} x\left(x^{2}+a x+b\right)^{\frac{n-1}{n}} \\
& -\frac{n \alpha^{\frac{3 n-2}{n}}}{[2 \beta(3 n-2)+a(2 n-1)]}\left[\left(\frac{x-\beta}{\alpha}\right)^{2}\right. \\
& \left.+\frac{2 n}{2 n-1}\left(\frac{x-\beta}{\alpha}\right)+\frac{2 n}{2 n-1}\right]^{\frac{n-1}{n}}\left(\frac{x-\beta}{\alpha}-\frac{n}{n-1}\right)+C
\end{aligned}
$$

holds true, where $\alpha$ and $\beta$ are given by (78) and (79), respectively.

Proof. It is easy to see, after integrating by parts, that

$$
\int \sqrt[n]{\left(x^{2}+a x+b\right)^{n-1}} d x=x\left(x^{2}+a x+b\right)^{\frac{n-1}{n}}-\frac{n-1}{n} \int \frac{\left(2 x^{2}+a x\right)}{\sqrt[n]{x^{2}+a x+b}} d x
$$

which is equivalent to

$$
\int \frac{x^{2}+\frac{2 n-1}{3 n-2} a x+b \cdot \frac{n}{3 n-2}}{\sqrt[n]{x^{2}+a x+b}} d x=\frac{n}{3 n-2} x\left(x^{2}+a x+b\right)^{\frac{n-1}{n}}
$$


From (92), we obtain

$$
\int \frac{(x-\beta)^{2}+\left(2 \beta+a \frac{2 n-1}{3 n-2}\right) x+\left(\frac{n}{3 n-2} b-\beta^{2}\right)}{\sqrt[n]{x^{2}+a x+b}} d x=\frac{n x\left(x^{2}+a x+b\right)^{\frac{n-1}{n}}}{(3 n-2)}
$$

where $\beta$ is given as (79). In terms of (93), it then follows that

$$
\int \frac{x+\frac{n b-(3 n-2) \beta^{2}}{2 \beta(3 n-2)+a(2 n-1)}}{\sqrt[n]{x^{2}+a x+b}} d x=\frac{n x\left(x^{2}+a x+b\right)^{\frac{n-1}{n}}}{2 \beta(3 n-2)+a(2 n-1)}-\frac{3 n-2}{2 \beta(3 n-2)+a(2 n-1)} \int \frac{(x-\beta)^{2}}{\sqrt[n]{x^{2}+a x+b}} d x
$$

Combining (86) with (94), we get the desired equality (90).

Lemma 13. The following statements are equivalents

(a) $\theta=\frac{n \cdot\left(\theta^{2}+a \theta+b\right)-\beta^{2}(3 n-2)}{2 \beta(3 n-2)+(2 \theta+a)(2 n-1)}$,
(b) $\theta=\frac{-[(n-1) a+(6 n-4) \beta] \pm \sqrt{\Delta}}{2(3 n-2)}$,

where $\beta$ is given by (79) and $\Delta$, by

$$
\Delta=(n-1)^{2} a^{2}+2(n-1)(6 n-4) a \beta+4 n b(3 n-2)
$$

Proof.

$$
\begin{aligned}
\theta & =\frac{n \cdot\left(\theta^{2}+a \theta+b\right)-\beta^{2}(3 n-2)}{2 \beta(3 n-2)+(2 \theta+a)(2 n-1)} \\
& \Leftrightarrow(6 n-4) \beta \theta+(4 n-2) \theta^{2}+a(2 n-1) \theta=n\left(\theta^{2}+a \theta+b\right)-\beta^{2}(3 n-2) \\
& \Leftrightarrow(3 n-2) \theta^{2}+[(n-1) a+(6 n-4) \beta] \theta+\left[\beta^{2}(3 n-2)-n b\right]=0 \\
& \Leftrightarrow \theta=\frac{-[(n-1) a+(6 n-4) \beta] \pm \sqrt{\Delta}}{2(3 n-2)}
\end{aligned}
$$

as required.

Lemma 14. The equality

$$
\int \frac{x}{\sqrt[n]{x^{2}+a x+b}} d x=p_{2}(x)+C
$$

is valid, where

$$
\begin{aligned}
p_{2}(x)= & \frac{n}{2 \beta(3 n-2)+(2 \theta+a)(2 n-1)}(x-\theta)\left[(x-\theta)^{2}+(2 \theta+a)(x-\theta)\right. \\
& \left.+\left(\theta^{2}+a \theta+b\right)\right]^{\frac{n-1}{n}}-\frac{n \alpha^{\frac{3 n-2}{n}}}{[2 \beta(3 n-2)+(2 \theta+a)(2 n-1)]}\left[\left(\frac{x-\theta-\beta}{\alpha}\right)^{2}\right. \\
& \left.+\frac{2 n}{2 n-1}\left(\frac{x-\theta-\beta}{\alpha}\right)+\frac{2 n}{2 n-1}\right]^{\frac{n-1}{n}}\left(\frac{x-\theta-\beta}{\alpha}-\frac{n}{n-1}\right)
\end{aligned}
$$

$\alpha, \beta$ and $\theta$ are given by (78), (79) and (97), respectively.

Proof. From Lemma 13, we deduce that

$$
\theta=\frac{n \cdot\left(\theta^{2}+a \theta+b\right)-\beta^{2}(3 n-2)}{2 \beta(3 n-2)+(2 \theta+a)(2 n-1)}
$$


which is equivalent to

$$
t+\theta=t+\frac{n \cdot\left(\theta^{2}+a \theta+b\right)-\beta^{2}(3 n-2)}{2 \beta(3 n-2)+(2 \theta+a)(2 n-1)}
$$

Combining the change of variable

$$
x=t+\theta,
$$

with (101), we deduce that

$$
\begin{aligned}
\int \frac{x}{\sqrt[n]{x^{2}+a x+b}} d x & =\int \frac{t+\theta}{\sqrt[n]{t^{2}+(2 \theta+a) t+\left(\theta^{2}+a \theta+b\right)}} d t \\
& =\int \frac{t+\frac{n \cdot\left(\theta^{2}+a \theta+b\right)-\beta^{2}(3 n-2)}{2 \beta(3 n-2)+(2 \theta+a)(2 n-1)}}{\sqrt[n]{t^{2}+(2 \theta+a) t+\left(\theta^{2}+a \theta+b\right)}} d t
\end{aligned}
$$

By using (90) and (103) as well as (102), we have (98).

We now give the proof of Proposition 3

Proof. Combining definition 1 with the lemma 14, we obtain 74.

Proposition 4. Let be $u(x)=\sqrt[n]{x^{2}+a x+b}$. Then the Caputo-Fabrizio fractional integral of $u(x)$ is given by

$$
I_{a x}^{\alpha} u(x)=(1-\alpha) \cdot u(x)+\alpha \cdot\left[p_{5}(x)-p_{4}(x)\right]+C,
$$

where $p_{4}(x)$ y $p_{5}(x)$ are given by (71) and (106), respectively.

To proof Proposition 4, we need the following lemmas

Lemma 15. The equality

$$
\int(x+1) \sqrt[n]{x^{2}+a x+b} d x=p_{5}(x)+C
$$

holds true, where

$$
\begin{aligned}
p_{5}(x)= & \frac{n}{2 \beta(3 n+2)+(2 \theta+a-2)(2 n+1)}(x+1-\theta)\left[(x+1-\theta)^{2}\right. \\
& \left.+(2 \theta+a-2)(x+1-\theta)+\left(\theta^{2}+(a-2) \theta+(b-a+1)\right)\right]^{\frac{n+1}{n}} \\
& -\frac{n \alpha^{\frac{3 n+2}{n}}}{[2 \beta(3 n+2)+(2 \theta+a-2)(2 n+1)]}\left[\left(\frac{x+1-\theta-\beta}{\alpha}\right)^{2}\right. \\
& \left.+\frac{2 n}{2 n+1}\left(\frac{x+1-\theta-\beta}{\alpha}\right)+\frac{2 n}{2 n+1}\right]^{\frac{n+1}{n}}\left(\frac{x+1-\theta-\beta}{\alpha}-\frac{n}{n+1}\right)
\end{aligned}
$$

$\alpha, \beta$ and $\theta$ are given by (55), (56) and (69), respectively.

Proof. By using the change of variable

$$
x+1=t
$$

we obtain

$$
\int(x+1) \sqrt[n]{x^{2}+a x+b} d x=\int t \sqrt[n]{t^{2}+(a-2) t+(b-a+1)} d t
$$

From (70) and (108) as well as (107), we obtain (105). We complete the proof. 
Lemma 16. The equality

$$
\int \sqrt[n]{x^{2}+a x+b} d x=p_{5}(x)-p_{4}(x)+C
$$

holds true, where $p_{4}(x)$ and $p_{5}(x)$ are given by (71) and (106), respectively.

Proof. It is easy to see that

$$
\int \sqrt[n]{x^{2}+a x+b} d x=\int(x+1) \sqrt[n]{x^{2}+a x+b} d x-\int x \sqrt[n]{x^{2}+a x+b} d x
$$

By (70) and (105) as well as (110), it is easy to see (109).

We now give the proof of Proposition 4

Proof. Combining definition 1 with the lemma 16, we obtain (104).

Proposition 5.Let be $u(x)=\frac{1}{\sqrt[n]{x^{2}+a x+b}}$. Then the Caputo-Fabrizio fractional integral of $u(x)$ is given by

$$
I_{a x}^{\alpha} u(x)=(1-\alpha) \cdot u(x)+\alpha \cdot\left[p_{6}(x)-p_{2}(x)\right]+C,
$$

where $p_{2}(x)$ y $p_{6}(x)$ are given by (99) and (113), respectively.

To proof Proposition 5, we need the following lemmas

Lemma 17. The equality

$$
\int \frac{x+1}{\sqrt[n]{x^{2}+a x+b}} d x=p_{6}(x)+C
$$

is valid, where

$$
\begin{aligned}
p_{6}(x)= & \frac{n}{2 \beta(3 n-2)+(2 \theta+a-2)(2 n-1)}(x+1-\theta)\left[(x+1-\theta)^{2}\right. \\
& \left.+(2 \theta+a-2)(x+1-\theta)+\left(\theta^{2}+(a-2) \theta+b-a+1\right)\right]^{\frac{n-1}{n}} \\
& -\frac{n \alpha^{\frac{3 n-2}{n}}}{[2 \beta(3 n-2)+(2 \theta+a-2)(2 n-1)]}\left[\left(\frac{x+1-\theta-\beta}{\alpha}\right)^{2}\right. \\
& \left.+\frac{2 n}{2 n-1}\left(\frac{x+1-\theta-\beta}{\alpha}\right)+\frac{2 n}{2 n-1}\right]^{\frac{n-1}{n}}\left(\frac{x+1-\theta-\beta}{\alpha}-\frac{n}{n-1}\right),
\end{aligned}
$$

$\alpha, \beta$ and $\theta$ are given by (78), (79) and (97), respectively.

Proof. By using the change of variable

$$
x+1=t
$$

we obtain

$$
\int \frac{x+1}{\sqrt[n]{x^{2}+a x+b}} d x=\int \frac{t}{\sqrt[n]{t^{2}+(a-2) t+(b-a+1)}} d t
$$

By using (98) and (115) as well as (114), we deduce (112).

Lemma 18. The equality

$$
\int \frac{1}{\sqrt[n]{x^{2}+a x+b}} d x=p_{6}(x)-p_{2}(x)+C
$$

holds true, where $p_{2}(x)$ and $p_{6}(x)$ are given by (99) and (113), respectively. 
Proof. It is easy to see that

$$
\int \frac{1}{\sqrt[n]{x^{2}+a x+b}} d x=\int \frac{x+1}{\sqrt[n]{x^{2}+a x+b}} d x-\int \frac{x}{\sqrt[n]{x^{2}+a x+b}} d x
$$

By using (112) and (98) as well as (117), we deduce (116).

We now give the proof of Proposition 5

Proof. Combining definition 1 with the lemma 18, we obtain (111).

Proposition 6. Let be $u(x)=\frac{x^{2}}{\sqrt[n]{x^{2}+a x+b}}$. Then the Caputo-Fabrizio fractional integral of $u(x)$ is given by

$$
I_{a x}^{\alpha} u(x)=(1-\alpha) \cdot u(x)+\alpha \cdot\left[p_{1}(x)+\left(2 \beta+\beta^{2}\right) p_{2}(x)-\beta^{2} p_{6}(x)\right]+C
$$

where $p_{1}(x), p_{2}(x)$ y $p_{6}(x)$ are given by (87), (99) and (113), respectively.

To proof Proposition 6, we need the following lemma

Lemma 19.The equality

$$
\int \frac{x^{2} d x}{\sqrt[n]{x^{2}+a x+b}}=p_{1}(x)+\left(2 \beta+\beta^{2}\right) p_{2}(x)-\beta^{2} p_{6}(x)+C .
$$

holds true, where $p_{1}(x), p_{2}(x)$ and $p_{6}(x)$ are given by (87), (99) and (113), respectively.

Proof. It is easy to see that

$$
\int \frac{x^{2} d x}{\sqrt[n]{x^{2}+a x+b}}=\int \frac{(x-\beta)^{2} d x}{\sqrt[n]{x^{2}+a x+b}}+2 \beta \int \frac{x d x}{\sqrt[n]{x^{2}+a x+b}}-\beta^{2} \int \frac{d x}{\sqrt[n]{x^{2}+a x+b}}
$$

In terms of (86) and (98) as well as (116), we deduce equality (119).

We now give the proof of Proposition 6.

Proof. Combining definition 1 with the lemma 19, we obtain (118).

Proposition 7.Let be $u(x)=x^{2} \cdot \sqrt[n]{x^{2}+a x+b}$. Then the Caputo-Fabrizio fractional integral of $u(x)$ is given by

$$
I_{a x}^{\alpha} u(x)=(1-\alpha) \cdot u(x)+\alpha \cdot\left[p_{3}(x)+\left(2 \beta+\beta^{2}\right) p_{4}(x)-\beta^{2} \cdot p_{5}(x)\right]+C,
$$

where $p_{3}(x), p_{4}(x)$ y $p_{5}(x)$ are given by (63), (71) y (106), respectively.

To proof Proposition 7, we need the following lemma

Lemma 20. The equality

$$
\int x^{2} \sqrt[n]{x^{2}+a x+b} d x=p_{3}(x)+\left(2 \beta+\beta^{2}\right) p_{4}(x)-\beta^{2} \cdot p_{5}(x)+C
$$

holds true, where $p_{3}(x), p_{4}(x)$ and $p_{5}(x)$ are given by (63), (71) and (106), respectively.

Proof. It is easy to see that

$$
\int x^{2} \sqrt[n]{x^{2}+a x+b} d x=\int(x-\beta)^{2} \cdot \sqrt[n]{x^{2}+a x+b} d x+2 \beta \cdot \int x \cdot \sqrt[n]{x^{2}+a x+b} d x-\beta^{2} \cdot \int \sqrt[n]{x^{2}+a x+b} d x
$$

From (62) and (70) as well as (109), equality (121) follows.

We now give the proof of Proposition 7.

Proof. Combining definition 1 with the lemma 20, we obtain (120). 


\section{Fractional derivative of Caputo-Fabrizio for some elementary functions}

Here, we consider some examples on Caputo-Fabrizio Fractional Derivative

Theorem 9. Let $a, b, \alpha \in \mathbb{R}$ such that $0<\alpha<1$. Then

$$
D_{a t}^{\alpha} e^{b t}=\frac{b}{\alpha+(1-\alpha) b}\left(e^{b t}-e^{-\frac{\alpha}{1-\alpha} t} e^{\frac{\alpha+(1-\alpha) b}{1-\alpha} a}\right)
$$

Proof. From definition 2, we have

$$
\begin{aligned}
D_{a t}^{\alpha} e^{b t} & =\frac{1}{1-\alpha} \int_{a}^{t} e^{-\frac{\alpha}{1-\alpha}(t-s)} b e^{b s} d s \\
& =\frac{b}{\alpha+(1-\alpha) b}\left(e^{b t}-e^{-\frac{\alpha}{1-\alpha} t} e^{\frac{\alpha+(1-\alpha) b}{1-\alpha} a}\right) .
\end{aligned}
$$

This completes the proof.

Theorem 10. Let $a, \alpha \in \mathbb{R}$ such that $0<\alpha<1$. Then the equality

$$
D_{a t}^{\alpha}(t-a)^{n}=\sum_{i=1}^{n}(-1)^{i-1} \frac{(1-\alpha)^{i-1}}{\alpha^{i}} \frac{n !}{(n-i) !}(t+a)^{n-i}+(-1)^{n} \frac{n !}{\alpha^{n}}(1-\alpha)^{n-1} e^{-\frac{\alpha}{1-\alpha}(t-a)}
$$

holds.

Proof. We will use the principle of mathematical induction. Let $P(n)$ be

$$
P(n) \equiv D_{a t}^{\alpha}(t-a)^{n}=\sum_{i=1}^{n}(-1)^{i-1} \frac{(1-\alpha)^{i-1}}{\alpha^{i}} \frac{n !}{(n-i) !}(t+a)^{n-i}+(-1)^{n} \frac{n !}{\alpha^{n}}(1-\alpha)^{n-1} e^{-\frac{\alpha}{1-\alpha}(t-a)}
$$

For our base case, we need to show $P(1)$ is true, meaning that

$$
D_{a t}^{\alpha}(t-a)=\sum_{i=1}^{1}(-1)^{i-1} \frac{(1-\alpha)^{i-1}}{\alpha^{i}} \frac{1 !}{(1-i) !}(t-a)^{1-i}+(-1)^{1} \frac{1 !}{\alpha^{1}}(1-\alpha)^{1-1} e^{-\frac{\alpha}{1-\alpha}(t-a)} .
$$

This is trivial, since

$$
\begin{aligned}
D_{a t}^{\alpha}(t-a) & =\frac{1}{1-\alpha} \int_{a}^{t} e^{-\frac{\alpha}{1-\alpha}(t-s)}(s-a)^{\prime} d s \\
& =\frac{1}{\alpha}-\frac{1}{\alpha} e^{-\frac{\alpha}{1-\alpha}(t-a)} \\
& =\sum_{i=1}^{1}(-1)^{i-1} \frac{(1-\alpha)^{i-1}}{\alpha^{i}} \frac{1 !}{(1-i) !}(t-a)^{1-i}+(-1)^{1} \frac{1 !}{\alpha^{1}}(1-\alpha)^{1-1} e^{-\frac{\alpha}{1-\alpha}(t-a)}
\end{aligned}
$$

For the inductive step, assume that for some $n, P(n)$ holds, so

$$
D_{a t}^{\alpha}(t-a)^{k}=\sum_{i=1}^{k}(-1)^{i-1} \frac{(1-\alpha)^{i-1}}{\alpha^{i}} \frac{k !}{(k-i) !}(t-a)^{k-i}+(-1)^{k} \frac{k !}{\alpha^{k}}(1-\alpha)^{k-1} e^{-\frac{\alpha}{1-\alpha}(t-a)} .
$$


We need to show that $P(n+1)$ holds, meaning that

$$
\begin{aligned}
D_{a t}^{\alpha}(t-a)^{k+1} & =\frac{1}{1-\alpha} \int_{a}^{t} e^{-\frac{\alpha}{1-\alpha}(t-s)}\left((s-a)^{k+1}\right)^{\prime} d s \\
& =\frac{k+1}{1-\alpha} \int_{a}^{t} e^{-\frac{\alpha}{1-\alpha}(t-s)}(s-a)^{k} d s \\
& =\frac{k+1}{\alpha}(t-a)^{k}-\frac{(k+1)(1-\alpha)}{\alpha} D_{a t}^{\alpha}(t-a)^{k} .
\end{aligned}
$$

To see this, note that

$$
\begin{aligned}
D_{a t}^{\alpha}(t-a)^{k+1}= & \frac{k+1}{\alpha}(t-a)^{k}-\frac{(k+1)(1-\alpha)}{\alpha} \sum_{i=1}^{k}(-1)^{i-1} \frac{(1-\alpha)^{i-1}}{\alpha^{i}} \frac{k !}{(k-i) !}(t \\
& -a)^{k-i}-\frac{(k+1)(1-\alpha)}{\alpha}(-1)^{k} \frac{k !}{\alpha^{k}}(1-\alpha)^{k-1} e^{-\frac{\alpha}{1-\alpha}(t-a)} \\
= & \frac{k+1}{\alpha}(t-a)^{k}+\sum_{i=1}^{k}(-1)^{i} \frac{(1-\alpha)^{i}}{\alpha^{i+1}} \frac{(k+1) k !}{(k-i) !}(t \\
& -a)^{k-i}+(-1)^{k+1} \frac{(k+1) !}{\alpha^{k+1}}(1-\alpha)^{k} e^{-\frac{\alpha}{1-\alpha}(t-a)} \\
= & \frac{k+1}{\alpha}(t-a)^{k}+\sum_{i=2}^{k+1}(-1)^{i-1} \frac{(1-\alpha)^{i-1}}{\alpha^{i}} \frac{(k+1) !}{(k+1-i) !}(t \\
& -a)^{k+1-i}+(-1)^{k+1} \frac{(k+1) k !}{\alpha^{k+1}}(1-\alpha)^{k} e^{-\frac{\alpha}{1-\alpha}(t-a)} \\
= & \sum_{i=1}^{k+1}(-1)^{i-1} \frac{(1-\alpha)^{i-1}}{\alpha^{i}} \frac{(k+1) !}{(k+1-i) !}(t-a)^{k+1-i} \\
& +(-1)^{k+1} \frac{(k+1) !}{\alpha^{k+1}(1-\alpha)^{k+1-1} e^{-\frac{\alpha}{1-\alpha}(t-a)} .}
\end{aligned}
$$

Thus $P(n+1)$ holds when $P(n)$ is true, so $P(n)$ is true for all natural numbers $n$.

Theorem 11. Let $n \in \mathbb{N}, \alpha, a, b \in \mathbb{R}$ such that $0<\alpha<1$. Then

$$
D_{a t}^{\alpha}(t-a)^{n} e^{b t}= \begin{cases}\frac{1}{1-\alpha} A_{1}(t)+\frac{b}{1-\alpha} B_{1}(t), & n=1 \\ \frac{1}{1-\alpha} A_{n}(t)+\frac{b}{1-\alpha} B_{n}(t), & n>1\end{cases}
$$

where $A_{1}(t), A_{n}(t), B_{1}(t)$ and $B_{n}(t)$ are given by (6), (7), (13) and (14), respectively.

Proof. From definition 2, we have

$$
\begin{aligned}
D_{a t}^{\alpha}(t-a)^{n} e^{b t} & =\frac{1}{1-\alpha} \int_{a}^{t} e^{\frac{-\alpha}{1-\alpha}(t-\tau)}\left[(\tau-a)^{n} e^{b \tau}\right]^{\prime} d \tau \\
& =\frac{n}{1-\alpha} \int_{a}^{t} e^{\frac{-\alpha}{1-\alpha} t+\frac{\alpha+(1-\alpha) b}{1-\alpha} \tau}(\tau-a)^{n-1} d \tau+\frac{b}{1-\alpha} \int_{a}^{t} e^{\frac{-\alpha}{1-\alpha} t+\frac{\alpha+(1-\alpha) b}{1-\alpha} \tau}(\tau-a)^{n} d \tau .
\end{aligned}
$$

In terms of (5) and (12), we obtain (122).

\section{Conclusion}

In this paper author has studied some theoretical properties concerning the Caputo-Fabrizio fractional derivative. Also composition of fractional operators has been obtained. In the same line it is given explicit formulas for Caputo-Fabrizio 
fractional operators of some elementary functions. To obtain such formulas, an auxiliary calculation in form of lemmas has been presented. As a future work, author is planning to use the properties presented in this work to analyse the qualitative properties of some Caputo-Fabrizio ordinary fractional differential equations.

\section{Acknowledgements}

This work is partially supported by UNGE, Universidad Nacional de Guinea Ecuatorial, and Havana University. The author would like to thank the professors Mariano Rodríguez Ricard and Ángela León Mecías as well as the anonymous referee for a careful checking of the details and for helpful comments that improved this paper.

\section{Competing interests}

The authors declare that they have no competing interests.

\section{Authors' contributions}

All authors have contributed to all parts of the article. All authors read and approved the final manuscript.

\section{References}

[1] Michele Caputo and Mauro Fabrizio, A new Definition of Fractional Derivative without Singular Kernel. Progr. Fract. Differ. Appl.(2015), vol. 1, No.2, pp. 73-77.

[2] Jorge Losada and Juan J. Nieto, Properties of a New Fractional Derivative without Singular Kernel. Progr. Fract. Differ. Appl.(2015), vol. 1, No. 2, pp. 87-89.

[3] Nasser Al-Salti, Erkinjon Karimov and Kishin Sadarangani, On a Differential Equation with Caputo-Fabrizio Fractional Derivative of Order $1<\beta \leq 2$ and Application to Mass-Spring-Damper System. Progr. Fract. Differ. Appl.(2016), vol. 2, No. 4, pp. $257-258$.

[4] J.F. Gómez-Aguilar, T. Córdova-Fraga, J.E. Escalante-Martínez, C. Calderón-Ramón, and R.F. Escobar-Jiménez, Electrical circuits described by a fractional derivative with regular Kernel. Revista Mexicana de Física (2016), vol. 62, pp. 144-145.

[5] Feng Gao and Xiao-Jun Yang, Fractional Maxwell Fluid with Fractional Derivatives Without Singular Kernel. THERMAL SCIENCE (2016), Vol. 20, pp. 871-872.

[6] Abdon Atangana and Dumitru Baleanu, New Fractional Derivatives with Nonlocal and Non-Singular Kernel: Theory and Application to Heat Transfer Model. THERMAL SCIENCE (2016), pp.1-2.

[7] Zhanbing Bai and Haishen Lü, Positive solutions for boundary value problem of nonlinear fractional differential equation. J. Math. Anal. Appl.(2005), vol. 311, pp. 495-496.

[8] Michele Caputo and Mauro Fabrizio, Applications of New Time and Spatial Fractional Derivatives with Exponential Kernels. Progr. Fract. Differ. Appl.(2016), vol. 2, No. 1, pp. 1-11.

[9] S. Etemad and Sh. Rezapour, On a Two-Variables Fractional Partial Differential Inclusion Via Riemann-Liouville Derivative. Novi Sad J. Math.(2016), Vol. 46, pp. 45-46.

[10] Abdon Atangana and Ilknur Koca, On the new fractional derivative and application to nonlinear Baggs and Freedman model. J. Nonlinear Sci. Appl.(2016), vol. 9, pp. 2467-2469.

[11] Xiao-Jun Yang, Hari M. Srivastava and J. A. Tenreiro Machado, A New Fractional Derivative Without Singular Kernel. Application to the Modelling of the Steady Heat Flow, THERMAL SCIENCE (2016), Vol. 20, No. 2, pp. 753-754.

[12] Nasser Al-Salti, Erkinjon Karimov and Sebti Kerbal, Boundary-value problems for fractional heat equation involving CaputoFabrizio derivative. NTMSCI (2016), vol. 4, No. 4, pp. 79-80.

[13] Rubayyi T. Alqahtani, Atangana-Baleanu derivative with fractional order applied to the model of groundwater within an unconfined aquifer. J. Nonlinear Sci. Appl.(2016), vol. 9 , pp. 3647-3649. 
[14] Jagdev Singh, A new analysis for fractional rumor spreading dynamical model in a social network with Mittag-Leffler law. Chaos (2019), Vol.29, pp. 1-3.

[15] Jagdev Singh, Devendra Kumar and Dumitru Baleanu, On the analysis of fractional diabetes model with exponential law. Advances in Difference Equations (2018), https://doi.org/10.1186/s13662-018-1680-1, pp. 1-4.

[16] Jagdev Singh et al., New aspects of fractional Biswas-Milovic model with Mittag-Leffler. Math. Model. Nat. Phenom.(2019), Vol. 14, pp. 1-4.

[17] Sunil Dutt Purohit et al., A Hybrid analytical algorithm for nonlinear fractional wave-like equations. Math. Model. Nat. Phenom.(2019), Vol. 14, pp.1-4.

[18] Devendra Kumar et al., A new fractional SIRS-SI malaria disease model with application of vaccines, antimalarial drugs, and spraying. Advances in Difference Equations (2019), https://doi.org/10.1186/s13662-019-2199-9, pp. 1-3.

[19] Leah Edelstein-Keshet et al., Integral Calculus with Applications to the Life Sciences. February 26, 2014.

[20] Gorosko O.A., Hedrih (Stevanovic) K., The construction of the Lagrange Mechanics of the discrete hereditary systems, Facta Universitatis, Series: Mechanics, Automatic Control and Robotics (2007), Vol. 6, No 1, pp. 175 -176.

[21] Hedrih A., Mechanical models of the double DNA. International Journal of Medical Engineering and Informatics (2011), Vol. 3, No.4, pp. 394-410.

[22] Hedrih (Stevanovic) K., Dynamics of coupled systems, Nonlinear Analysis: Hybrid Systems (2008), Volume 2, pp.310-334.

[23] Gorosko O. A. i Hedrih (Stevanovic) K., Analiticka dinamika (mehanika) diskretnih naslednih sistema, (Analytical Dynamics (Mechanics) of Discrete Hereditary Systems), Monograph, p. 426, YU ISBN 86-7181-054-2,2001.

[24] Mihailo Lazarevi et.al., Advanced Topics on Applications of Fractional Calculus on Control Problems, System Stability and Modeling, Published by WSEAS Press (2014)

[25] Goyal S. and Perkins N.C., Looping mechanics of rods and DNA with non-homogeneous and discontinuous stiffness, Int J NonLinear Mech.(2008), Vol. 43, No. 10, pp.1121-1128.

[26] M. Caputo and M. Fabrizio, A new definition of fractional derivative without singular kernel Progr. Fract. Differ. Appl. (2015), Vol. 1, No. 2, pp.

[27] I. Podlubny, Fractional differential equations. Mathematics in Science and Engineering (1999), Vol. 198, pp.18-20.

[28] Anselmi C., Desantis P. and Scipioni A. Nanoscale echanical and dynamical properties of DNA single molecules, Biophys Chem.(2005), Vol. 113, pp.209-221

[29] Dr. Emad A. Al-Sabawi, Abdulaziz H. Marie, Image Deblurring Based FractionalOrder Differentiation and Genetic Algorithm (2017), Vol.7, pp.19-26.

[30] Panumart Sawangtong et al., An Analysis on the Fractional Asset Flow Differential Equations. Mathematics 2017, 5, 33; doi:10.3390/math5020033, pp. 1-3.

[31] Yi Zhang, Yifei Pu, Jiliu Zhou, Construction of Fractional differential Masks Based on Riemann-Liouville Definition. Journal of Computational Information Systems (2010), vol. 6, No.10, pp. 3191-3199.

[32] Rabha W. Ibrahim et al., Texture Enhancement for Medical Images Based on Fractional Differential Masks. Discrete Dynamics in Nature and Society (2013), Volume 2013, Article ID 618536, pp. 1-9.

[33] Chen Qing-li et al., A Fractional Differential Approach to Low Contrast Image Enhancement. International Journal of Knowledge and Language Processing (2012), Vol. 3, No. 2, pp. 20-27.

[34] Zhang Jun et al., A class of fractional-order multi-scale variational models and alternating projection algorithm for image denoising. Applied Mathematical Modelling (2011), Vol. 35 pp. 2516-2528.

[35] Lihong Huang et al., Adaptive fourth-order partial differential equation filter for image denoising. Applied Mathematics Letters (2011), Vol. 24, pp. 1282-1288.

[36] Marko Janev et al., Fully fractional anisotropic diffusion for image denoising. Mathematical and Computer Modelling (2011), Vol. 5, pp. 729-741.

[37] Vignesh Kothapalli, Shaveta Arora, Madasu Hanmandlu, Edge detection using fractional derivatives and information sets. Journal of Electronic Imaging (2018), vol. 27, p.1-10.

[38] P. Melchior et al., Fractional differentiation for edge detection. Signal Processing (2003), vol. 83, pp. 2421-2432.

[39] Chaobang Gao et al., Edge Detection Based on the Newton Interpolation's Fractional Differentiation. The International Arab Journal of Information Technology (2014), Vol. 11, No. 3, pp. 223-227.

[40] Reju John, Nissan Kunju, Detection of Alzhemier's Disease Using Fractional Edge Detection. IOSR Journal of VLSI and Signal Processing (2019), Vol. 9, pp. 1-5. 